\title{
Treatment of Combined Autoimmune Neutropenia and Immune Thrombocytopenia with Methotrexate
}

\author{
Maria Mitsogianni Nikolaos Mitsimponas Sabine Haase Aristoteles Giagounidis
}

Clinic for Oncology, Hematology and Palliative Care, Marien Hospital Düsseldorf, Düsseldorf, Germany

Dear Editor,

Combined autoimmune neutropenia (AIN) and immune thrombocytopenia (ITP) is an extremely rare and therapeutically challenging disease [1]. Patients may present with extremely low neutrophil and platelet counts, leading to repetitive infectious and bleeding complications [2,3]. We report the cases of 2 patients who achieved durable control of both conditions with long-term stabilization of the neutrophil and platelet count under treatment with methotrexate.

The first patient was a 36-year-old male with persistent neutropenia and thrombocytopenia, who presented with recurrent skin infections. Anti-neutrophil antibodies were detected through a combination of indirect granulocyte immunofluorescence and granulocyte agglutination test, thereby supporting the presence of an autoimmune mechanism. Treatment with prednisolone led to normalization of the platelet count. However, application of G-CSF had no effect on the granulopoiesis despite multiple dose adjustments. Treatment was switched to methotrexate $15 \mathrm{mg} /$ week in combination with folic acid $5 \mathrm{mg} /$ week, resulting in a rise of the absolute neutrophil count above $1.0 \times 10^{9} / \mathrm{L} 4$ months later. Thirty-one months under low-dose methotrexate, peripheral blood counts remain stable, and there have been no further infectious complications. The second case refers to a 77-year-old female with progressive, combined, immune-mediated neutropenia and thrombocytopenia. The presence of antineutrophil antibodies was confirmed with the same method of indirect granulocyte immunofluorescence and granulocyte agglutination test. Based on patient preference, no G-CSF was applied for the treatment of neutropenia and, similarly to the first patient, a therapy with methotrexate and folic acid was initiated instead. Nine months under methotrexate, absolute neutrophil count rose above the neutropenic range, while the platelet count was also improved and stabilized above $100 \times 10^{9} / \mathrm{L}$. Fifteen months later, treatment is well-tolerated, and the patient remains asymptomatic.

Combined AIN and ITP is a very uncommon disease so that to date, very little data regarding therapeutic options and no widely accepted first-line treatment are available [1]. Stimulation with G-CSF has been established as an effective therapy for AIN [2]. Non response to G-CSF is in general very rare in patients with severe chronic neutropenia [4]. Nevertheless, this was the case in our first patient. Methotrexate is an immunosuppressive drug that has already shown efficacy in the treatment of AIN. In an observational study by Sicre de Fontbrune et al. [5] on adult patients with severe chronic primary neutropenia, immunosuppressive agents (mainly methylprednisolone, cyclophosphamide, and methotrexate) were effective in $44.4 \%$ of the cases and are being recommended by the authors for symptomatic patients who do not respond to G-CSF.

Myelosuppression is a known adverse effect of methotrexate. Still, in our report, the first patient maintained constant platelet counts during treatment, underscoring the benefit from the immunosuppressive effect of the drug. The second patient showed an increase of the platelet count parallel to the increase of the neutrophil count, demonstrating that, despite the known myelotoxicity, methotrexate can be an effective initial treatment for the immune thrombocytopenic portion of cytopenias as well.

To our knowledge, these patients are the first documented cases of successful treatment of combined AIN and ITP with methotrexate. Our report suggests that treatment with methotrexate can result in a long-term improvement of both neutrophil and platelet counts and good symptom control in combined AIN and ITP. Therefore, this treatment can be considered a valuable option for this group of patients.

\section{Statement of Ethics}

The authors state that subjects have given their written informed consent to publish their case.

\section{Disclosure Statement}

The authors have no conflicts of interest to declare.

\section{Funding Sources}

The authors did not receive any funding.

\section{KARGER}

(C) 2019 S. Karger AG, Basel
Maria Mitsogianni

Clinic for Oncology, Hematology and Palliative Care Marien Hospital Düsseldorf

Rochusstrasse 2, DE-40479 Düsseldorf (Germany)

E-Mail mitsogiannimaria @ gmail.com 


\section{References}

1 Martino R, Muñiz-Díaz E, Arilla M, Ibáñez M, Altés A, Guanyabens C, et al. Combined autoimmune cytopenias. Haematologica. 1995 Jul-Aug;80(4):305-10.

2 Afzal W, Owlia MB, Hasni S, Newman KA. Autoimmune neutropenia updates: etiology, pathology, and treatment. South Med J. 2017 Apr;110(4):300-7.
3 Neunert C, Lim W, Crowther M, Cohen A, Solberg L Jr, Crowther MA; American Society of Hematology. The American Society of Hematology 2011 evidence-based practice guideline for immune thrombocytopenia. Blood. 2011 Apr;117(16):4190-207.

4 Dale DC, Cottle TE, Fier CJ, Bolyard AA, Bonilla MA, Boxer LA, et al. Severe chronic neutropenia: treatment and follow-up of patients in the Severe Chronic Neutropenia International Registry. Am J Hematol. 2003 Feb; 72(2):82-93.
5 Sicre de Fontbrune F, Moignet A, Beaupain B, Suarez F, Galicier L, Socié G, et al.; French Severe Chronic Neutropenia Registry. Severe chronic primary neutropenia in adults: report on a series of 108 patients. Blood. 2015 Oct; 126(14):1643-50. 